\title{
ФОРМИРОВАНИЕ СТРАТЕГИЧЕСКОГО ПОРТФЕЛЯ ПРОЕКТОВ В ОРГАНИЗАЦИЯХ ТЕРМИНАЛЬНОЙ ДОСТАВКИ ГРУЗОВ АВТОМОБИЛЬНЫМ ТРАНСПОРТОМ
}

\begin{abstract}
В статье предложены методические основы к разработке моделей комплексного обоснования приоритетности проектов стратегического портфеля систем перевозок в организациях терминальной доставки грузов автомобильным транспортом, который предусматривает оценку проектов по параметрам стратегической значимости и финансовой эффективности.
\end{abstract}

\section{1. ВВЕДЕНИЕ}

При формировании стратегически ориентированного (стратегического) портфеля, или просто портфеля, поскольку, по определению [2, с. 121], портфель организации является нацеленным на достижение стратегических целей последней, одними из наиболее значимых выступают задачи идентификации, определения приоритетности и отбора стратегически ориентированных проектов в портфель.

Концептуально, известные на сегодняшний день методы и модели стратегически ориентированного управления портфелем могут быть разделены на две группы.

К первой группе можно отнести методы и модели, условия реализации которых не предусматривают, что процессы идентификации проектов интегрированы с процессами реализации стратегий. Проекты избираются фрагментарно, изолированно, как правило, на первом этапе, используя методы оценивания финансовой эффективности инвестиционных проектов и капитального бюджетирования. А затем, на втором этапе, устанавливается их соответствие (или несоответствие) стратегии развития организации. При этом вопрос формирования критериев соответствия является в данных методах достаточно проблематичным. Важным недостатком данных методов является то, что они реализуются в условиях, когда в организации отсутствуют системные постоянно действующие механизмы формирования стратегически ориентированного портфеля как средства реализации стратегии и привлечения к этому процессу всех структурных подразделений организации. Соответственно не исключается дублирование инвестиционных проектов и игнорирование эффекта синергии.

${ }^{1}$ Dr hab Tetiana Vorkut, profesor, Katedra Transportowego Prawa i Logistyki Narodowego Uniwersytetu Transportu w Kijowie, Ukraina.

${ }^{2}$ Dr Oksana Bilonog, Katedra Transportowego Prawa i Logistyki Narodowego Uniwersytetu Transportu w Kijowie, Ukraina. 
Вторая группа методов и моделей исходит из того, что процессы идентификации проектов интегрированы с процессами реализации стратегий. Таким образом, все проекты, идентифицированные с использованием этих методов, должны быть, по определению, стратегически ориентированными. Другой вопрос, что их стратегическая значимость не будет тождественной. Это предопределяется тем, что не будет тождественной, в общем случае, приоритетность стратегических целей и задач организации, на решение которых направлены данные проекты. Собственно, в качестве метода данной группы можно рассматривать метод сбалансированной системы показателей. Наиболее существенным недостатком методов данной группы, в частности, метода сбалансированной системы показателей, является то, что высокий вес, устанавливаемый, как правило, достаточно субъективно, предоставляется критерию стратегической значимости и существенно более низкий другим критериям, в частности финансовой эффективности [4, с. 155].

Таким образом, финансово высокоэффективные проекты могут оставаться за пределами портфеля и, в то же время, проекты с низкой финансовой эффективностью в состав такого портфеля войти. При этом цель высшего уровня для организаций бизнеса - максимизация благосостояния их владельцев. Когда стратегия, в итоге, не является ориентированной на данную цель, то может стоять вопрос о корректировке стратегии или, даже, разработке новой. Вместе с тем, важным является и корректная оценка выгод и затрат по проекту, в частности в контексте значимости последнего для реализации следующих проектов организации.

ЦЕЛЬ СТАТЬИ закЛючается в обосновании методических основ построения моделей комплексного обоснования приоритетности проектов стратегического портфеля систем перевозок организаций терминальной доставки грузов автомобильным транспортом (дальше - ТДГАТ) как основы для принятия управленческих решений относительно формирования последнего.

\section{2. ОСНОВНАЯ ЧАСТЬ}

Обоснованным кажется предположение, что как повышения эффективности процессов формирования стратегически ориентированного портфеля проектов, так и эффективности собственно самого портфеля проектов требует определения стратегически наиболее значимых задач (проблемных ситуаций), рис. 1. Если считать, что для организационных структур систем перевозок стратегические цели развития сформулированы, функции (свойства) в соответствии с целями определены, критерии согласованности со стратегическими целями избраны, а их целевые значения установлены, то можно оценить стратегическую значимость задач, решение которых должно способствовать достижению стратегических целей. Для этого можно использовать такой метод обоснования многокритериальных решений как метод анализа иерархий [8]. В соответствии с теоретическими принципами данного метода алгоритм оценки стратегической приоритетности задач, на решение которых направляются проекты и/или программы, можно представить следующим образом.

Во-первых, необходимо сформировать многоуровневую иерархическую структуру, которая позволила бы определить значимость задач, которые нуждаются в решении в условиях реализации системой перевозок организации ТДГАТ 
избранной стратегии развития. Алгоритм построения таких структур предусматривает, что на верхнем уровне (уровень 0) структуры расположен лишь один объект - интегрированный критерий, который можно разложить на несколько объектов - уровень 1, что следует непосредственно за 0 -м уровнем иерархии. Каждый объект 1-го уровня иерархии, в свою очередь, детализируется по нескольким объектам следующего уровня и т. д. На самом низком уровне иерархической структуры расположены объекты, которые анализируются и упорядочиваются по значимости. Построенная, исходя из данных рассуждений, многоуровневая иерархическая структура оценивания стратегической значимости задач систем перевозок представлена на рис. 2.

Во-вторых, необходимо установить относительные весовые коэффициенты для каждого объекта сформированной структуры в пределах каждого уровня иерархии. Для определения весовых коэффициентов в пределах целевого уровня предлагается использовать общеорганизационные приоритеты, функционального уровня приоритеты организационной структуры систем перевозок, см. рис. 2. Для определения относительных весовых коэффициентов может быть применен метод экспертного опроса, который предусматривает прохождение следующих этапов:

а) определение экспертов, которые должны быть привлечены к опросу. Для оценки значимости локальных стратегических целей, $G_{i j}^{n}$, и функций (свойств), $F_{i j}^{n}$, систем перевозок эксперты должны избираться из менеджеров более высокого и, частично, среднего уровня, а для оценки значимости задач, $O_{i j}^{n}$, какие должны быть решены для достижения поставленных стратегических целей - менеджеры соответствующих территориальных представительств и общеорганизационных функциональных подразделений организаций ТДГАТ;

б) выбор метода проведения опроса и разработки анкет. Исходя из цели опроса можно использовать метод групповых оценок. 


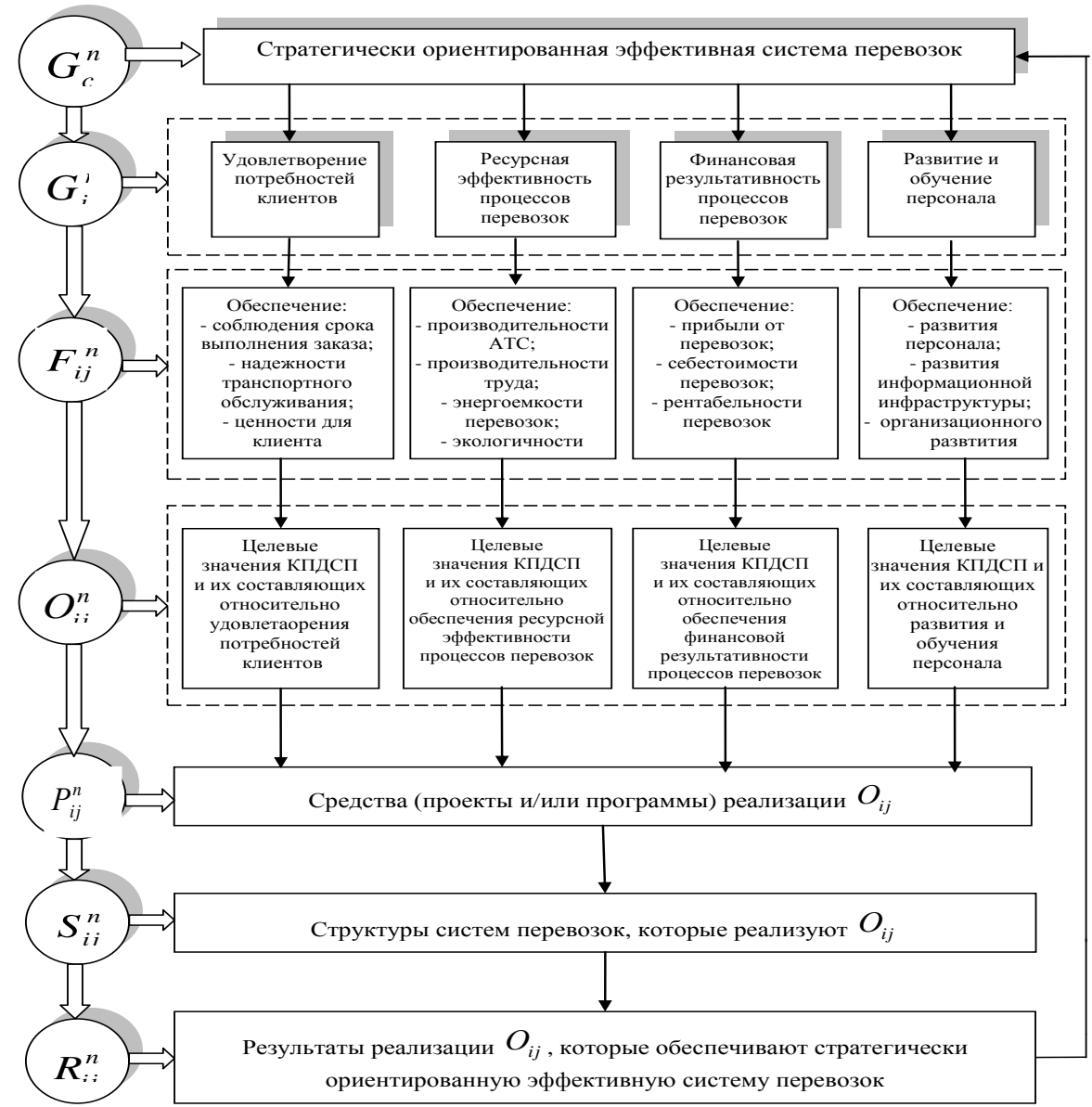

Рис. 1. Модель реализации стратегически ориентированной эффективной системы перевозок в организациях ТДГАТ 


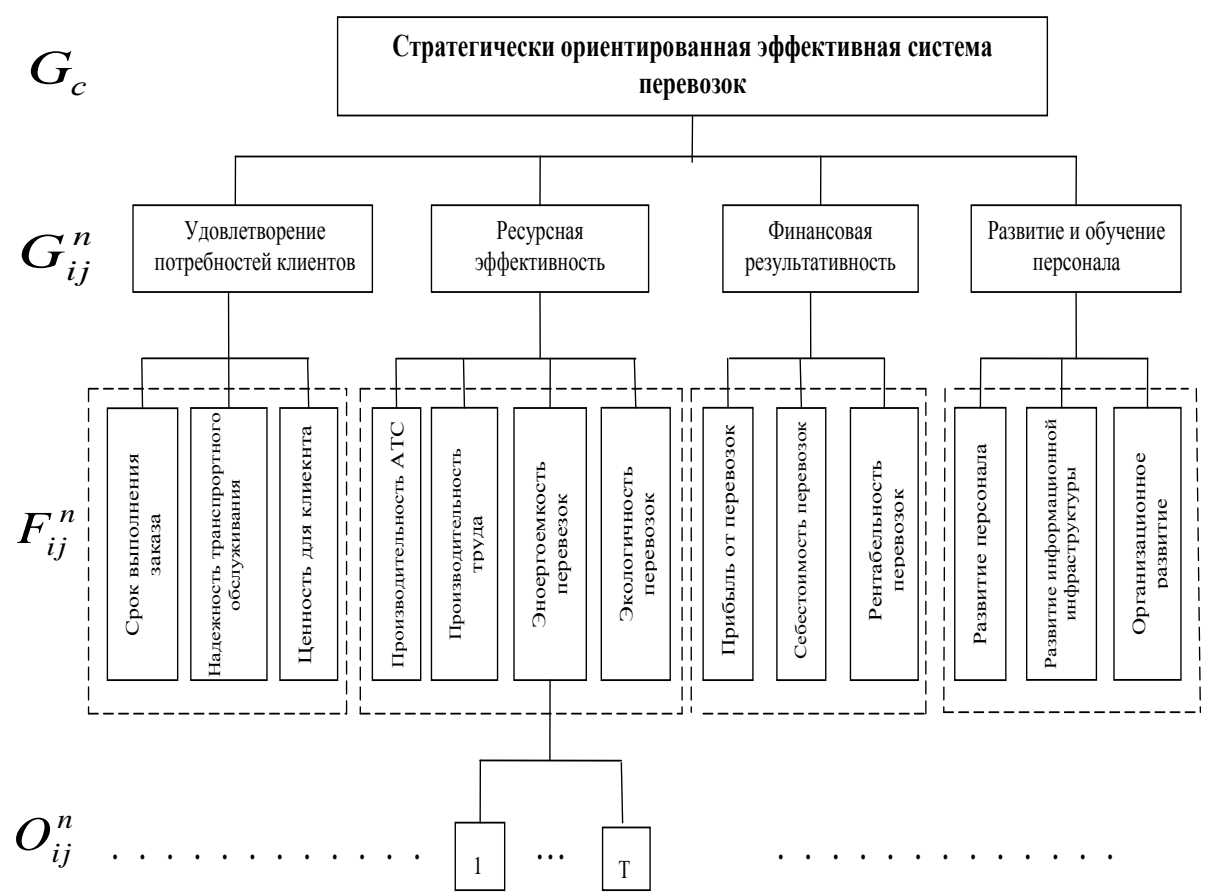

Рис. 2. К определению стратегической значимости задач в системах перевозок организаций ТДГАТ

Группам экспертов, которые привлекаются к опросу, предлагается заполнить матрицы попарных сравнений [1], которые отвечают уровням иерархии, вида:

$$
\mathrm{A}=\left(\begin{array}{cccc}
w_{1} / w_{1} & w_{1} / w_{2} & \ldots & w_{1} / w_{n} \\
w_{2} / w_{1} & w_{2} / w_{2} & \ldots & w_{2} / w_{n} \\
\ldots & \ldots & \ldots & \ldots \\
w_{n} / w_{1} & w_{n} / w_{2} & \ldots & w_{n} / w_{n}
\end{array}\right),
$$

где $w_{l}, w_{2}, \ldots, w_{n}$ - значимость (весовые коэффициенты) объектов, которые сопоставляются;

$n$ - количество объектов, которые сопоставляются.

в) проведение статистической обработки результатов опроса с целью определения значимости каждого объекта оценки на каждом из идентифицированных уровней иерархии.

При применении метода попарных сравнений важно достичь согласованности суждений. Как известно, идеальная матрица сравнений есть обратно-симметричной и согласованной. Столбик $\mathrm{w}=\left(\begin{array}{c}w_{1} \\ \ldots \\ w_{n}\end{array}\right)$ является собственным столбиком матрицы 
$\mathrm{A}=\left\|a_{i j}\right\|$ с собственным значением $\lambda=n, \mathrm{Aw}=n w$. Когда $\lambda_{\max } \neq n$ (всегда $\lambda_{\max } \geq n$ ), то за степень отклонения положительной обратно-симметричной матрицы от согласованной выступает индекс согласованности $-\frac{\lambda_{\max }-n}{n-1}$. Этот показатель указывает на степень приближенности соответствующей матрицы к согласованной. Считается, что когда индекс согласованности не превышает 0,1, то степень согласованности суждений является удовлетворительной [6].

На основании полученных на предыдущем этапе экспертной оценки количественных оценок могут быть вычислены значения весовых коэффициентов как среднее геометрическое для элементов матрицы $\mathrm{A}=\left\|a_{i j}\right\|$ :

$$
c_{i}=\left(\prod_{j=1}^{n} a_{j}\right)^{\frac{1}{n}}, i=1,2, \ldots, I,
$$

и, соответственно, определяются нормализованные весовые коэффициенты:

$$
w_{i}=\frac{c_{i}}{\sum_{i=1}^{n} c_{i}}, i=1,2, \ldots, I
$$

Нормализованные весовые коэффициенты образуют для каждой матрицы сравнений $\mathrm{A}=\left\|a_{i j}\right\|$ собственный столбик, по которому приближенно определяется $\lambda_{\max }$ и который, в то же время, выступает как вектор весовых коэффициентов $\mathrm{W}=\left(\begin{array}{c}w_{1} \\ w_{2} \\ \cdots \\ w_{n}\end{array}\right)$.

Вектор приоритетов, который определяет оценки (приоритетность) анализируемых объектов, - задач $\mathrm{w}^{\mathrm{s}}=\left(\begin{array}{c}w_{1}^{s} \\ w_{2}^{s} \\ \ldots \\ w_{T}^{s}\end{array}\right)$, можно получить осуществляя последовательное умножение матриц, столбиками которых являются векторы приоритетов соответствующих элементов определенного уровня с точки зрения элемента непосредственно высшего уровня иерархической структуры (с учетом их связей).

Чем более высоким, в итоге, оказывается показатель стратегической значимости задачи, тем, соответственно, более значимым является ее решение для достижения стратегических целей организации, и, в то же время, являются более значимыми проекты и/или программы, - направленные на решение данной задачи. 
Стратегическая значимость собственно проектов оценивается по степени, в которой они обеспечивают реализацию соответствующих задач, с использованием бальных оценок. При этом должны быть учтены внешние эффекты проектов, которые, в данном контексте, рассматриваются как такие, что способствуют или, напротив, стоят на пути решения других задач системы перевозок, решение которых условиями идентификации этих проектов предусмотрено не было. При данных условиях стратегическую значимость (ранг) проектов можно определить следующим образом:

$$
C W^{s}=R^{s}
$$

где C - матрица бальных оценок проектов относительно их влияния на решение задач системы перевозок;

$W^{s}$ - вектор стратегической значимости (приоритетности) задач систем перевозок;

$R^{s} \quad-$ вектор стратегической значимости (приоритетности) проектов стратегического портфеля систем перевозок.

Принимая во внимание влияние каждого $p$-го проекта системы перевозок, $p=1,2, \ldots, P$, на решение каждой задачи $t, t=1,2, \ldots, T$, выражение (4) можно записать в виде:

$$
\left(\begin{array}{ccccc}
c_{11} & \ldots & c_{1 t} & \ldots & c_{1 T} \\
\ldots & \ldots & \ldots & \ldots & \ldots \\
c_{p 1} & \ldots & c_{p t} & \ldots & c_{p T} \\
\ldots & \ldots & \ldots & \ldots & \ldots \\
c_{P 1} & \ldots & c_{P t} & \ldots & c_{P T}
\end{array}\right) \times\left(\begin{array}{c}
w_{1}^{s} \\
\ldots \\
w_{t}^{s} \\
\ldots \\
w_{T}^{S}
\end{array}\right)=\left(\begin{array}{c}
r_{1}^{s} \\
\ldots \\
r_{p}^{s} \\
\ldots \\
r_{P}^{S}
\end{array}\right)
$$

где $c_{p t}$ - средняя (по бальным оценкам экспертов) оценка $p$-го проекта за влиянием на решение $t$ - й задачи, $p=1,2, \ldots, P, t=1,2, \ldots, T$.

Вторым важным аспектом оценивания проектов и/или программ в условиях анализа целесообразности включения их в стратегический портфель системы перевозок является оценивание их финансовой эффективности.

Данный анализ может основываться на одном или нескольких методов, которые используются для оценивания финансовой эффективности инвестиционных проектов.

Вместе с тем, принятие организацией определенной стратегии развития отображается и в приоритетах относительно выбора методов (критериев) оценивания эффективности инвестиционных проектов. Таким образом, представляет интерес определение ранга проектов, направленных на решение стратегических задач, по критерию оценивания финансовой эффективности инвестиционных проектов, на основе которого должна определяться приоритетность последних. 
Проведенный сравнительный анализ недостатков и преимуществ методов оценивания финансовой эффективности инвестиционных проектов позволяет рекомендовать отразить в этом критерии такие показатели как чистая приведенная стоимость (дальше - NPV), внутренняя ставка дохода (дальше - IRR), индекс прибыльности (дальше - B/C), срок окупаемости (дальше - PBP) и стратегическая чистая приведенная стоимость (дальше $-\mathrm{NPV}_{\mathrm{s}}$ ).

Условия оценивания финансовой эффективности инвестиционных проектов позволяют количественно учитывать степень риска последних. Для этого могут, например, применяться методы корректирования, которыми предусматривается интеграция риска в методы оценки финансовой эффективности инвестиционных проектов. Это, в частности, метод эквивалента определенности и метод откорректированных на риск ставок дисконтирования [3].

Для оценивания степени риска отдельных проектов может быть также применен метод анализа сценариев. Обычно рассматривают базовый, оптимистичный и пессимистический сценарии. На основе оценки вероятности хода событий по проекту по каждому из трех сценариев может быть определено математическое ожидание критериев финансовой эффективности инвестиционных проектов [3].

Значения показателей, в зависимости от постановки задачи - с учетом риска или без, финансовой эффективности проектов, которые избраны как критерии, переводятся в бальные оценки. Дальше, с учетом приоритетности стратегической составляющей и составляющей финансовой эффективности, определяется ранг проекта.

Значимость (весовые коэффициенты) показателей оценки финансовой эффективности могут устанавливаться на основе подходов, аналогичных тем, которые предлагается использовать в данной работе для определения значимости задач стратегического развития систем перевозок. Вместе с тем, эксперты, которые привлекаются к опросу, могут избираться из менеджеров высшего уровня и менеджеров финансовых подразделений организации.

Совместный анализ проектов организационных структур системы перевозок организации ТДГАТ создает предпосылки к выявлению эффекта синергии и, соответственно, реформированию проектов, которые составляют соответствующую совокупность.

Проекты, идентифицированные как одним организационным структурным подразделением, так и разными, в том числе на разных иерархических уровнях организации, могут быть независимыми, дополняющими или замещающими. В условиях, когда инвестиционные проекты, направленные на реализацию стратегии организации, рассматривать в рамках единого (интегрального) стратегического портфеля (подпортфеля), как проекты-компоненты, то появляется задача установления характера взаимосвязи между данными компонентами.

Как известно, различают независимые и зависимые инвестиционные проекты. Проекты считаются независимыми, когда их NPV равняется NPV стратегического портфеля проектов, в единых рамках которого данные проекты как компоненты могут быть реализованы. Соответственно, инвестиционные проекты можно считать зависимыми, когда сумма их NPV отличается от NPV портфеля проектов. Зависимые инвестиционные проекты, в свою очередь, могут быть разделены на такие, которые взаимодействуют на условиях замещения и дополнения. Инвестиционные проекты считаются такими, что взаимодействуют на условиях 
замещения, когда их суммарная NPV в рамках стратегического портфеля проектов является больше, чем NPV стратегического портфеля проектов. Инвестиционные проекты считаются такими, что взаимодействуют на условиях дополнения, если их суммарная NPV является меньше, чем NPV стратегического портфеля проектов. То есть, в этом случае имеет место эффект синергии.

Вышеизложенные ситуации можно описать, представляя отношение независимости, замещения и дополнения в контексте приведенных выгод и затрат, которые ожидаются от стратегического портфеля проектов и отдельных проектовкомпонентов.

На рис. 3 в зависимости от характера отношений между выгодами и затратами представлены соотношения между NPV стратегического портфеля проектов и суммой NPV проектов, которые его составляют [7].

\begin{tabular}{|c|c|c|c|}
\hline $\begin{array}{l}\text { Взаимосвязь } \\
\text { по затратам }\end{array}$ & Независимые & Дополняющие & Замещуающие \\
\hline Независимые & $N P V_{u \mu m}=\sum_{i=1}^{n} N P V_{i}$ & $\left.N P V_{u н m}\right\rangle \sum_{i=1}^{n} N P V_{i}$ & $N P V_{\text {uнt }}\left\langle\sum_{i=1}^{n} N P V_{i}\right.$ \\
\hline Дополняющие & $\left.N P V_{\text {инт }}\right\rangle \sum_{i=1}^{n} N P V_{i}$ & $\left.N P V_{\text {инm }}\right\rangle \sum_{i=1}^{n} N P V_{i}$ & $\begin{array}{c}\text { Результат неопределен, } \\
\text { необходимы } \\
\text { дополнительные } \\
\text { расчеты }\end{array}$ \\
\hline Замещзаюшие & $N P V_{\text {uнm }}\left\langle\sum_{i=1}^{n} N P V_{i}\right.$ & $\begin{array}{c}\text { Результат неопределен, } \\
\text { необходимы } \\
\text { дополнительные } \\
\text { расчеты }\end{array}$ & $N P V_{u \mu m}\left\langle\sum_{i=1}^{n} N P V_{i}\right.$ \\
\hline
\end{tabular}

Рис. 3. Анализ отношений зависимости в стратегическом портфеле проектов систем перевозок

Вместе с тем, как отмечают исследователи [5, с. 71], окончательное решение относительно значимости проекта для компании часто базируется не столько на выводах относительно моделей оценивания значимости проектов, сколько на внутреннем чувстве руководителей, которые принимают во внимание множество дополнительных эвристических элементов решения, которые сложно формализировать.

При этих обстоятельствах представляет интерес построение матрицы принятия решений относительно включения проектов и/или программ в итоговый вариант стратегического портфеля систем перевозок, на основе полученных рангов относительно стратегической значимости инвестиционных проектов и значимости финансовой эффективности этих проектов (в том числе с учетом риска), рис. 4. 
Низкая финансовая эффективность проекта при его высокой стратегической значимости, см. рис. 4, блок 3 , может быть присущей проектам, реализация которых есть, так сказать, “вынужденной”, поскольку предопределяется необходимостью выполнения: требований законодательства - обеспечения безопасности производства, охраны окружающей среды; социальных стандартов - обеспечения условий труда, учебы и отдыха персонала и т.д.; условий продолжения деятельности организации, когда необходимо заменить оборудование, которое физически изношено или потеряно. К данной группе также могут быть отнесенные проекты, которые имеют стратегические перспективы, для выявления которых при проведении финансового анализа проекта должны быть применены соответствующие методы оценивания, - например, метод стратегической чистой приведенной стоимости, или вышеупомянутый проект целесообразно рассматривать как компонент сложных проектов.

Значимость проектов по финансовой эффективности

\begin{tabular}{|c|c|c|c|}
\hline & високая & средняя & низкая \\
\hline 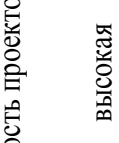 & $\begin{array}{l}1 \\
\text { Рекомендовать }\end{array}$ & $\begin{array}{l}2 \\
\text { Рекомендовать }\end{array}$ & $\begin{array}{c}\text { Требует } \\
\text { дальнейшего } \\
\text { рассмотрения }\end{array}$ \\
\hline 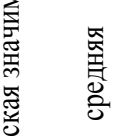 & $\begin{array}{l}4 \\
\text { Рекомендовать }\end{array}$ & $\begin{array}{c}5 \\
\text { Требует } \\
\text { дальнейшего } \\
\text { рассмотрения }\end{array}$ & $\begin{array}{l}6 \\
\text { рекомендовать }\end{array}$ \\
\hline 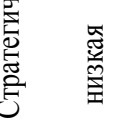 & $\begin{array}{c}7 \\
\text { Требует } \\
\text { дальнейшего } \\
\text { рассмотрения }\end{array}$ & $\begin{array}{l}8 \\
\text { рекомендовать }\end{array}$ & $\begin{array}{l}9 \\
\text { Некомендовать }\end{array}$ \\
\hline
\end{tabular}

Рис. 4. Матрица принятия решений относительно включения проекта в стратегический портфель систем перевозок

Вместе с тем, когда это масштабные проекты, с которыми связаны существенные, с точки зрения данной организации, капиталовложения, то это может указывать на необходимость корректирования стратегии в целом, или отдельных стратегических целей. Это же касается и масштабных, связанных с существенными капиталовложениями, проектов, которые имеют высокую финансовую эффективность, однако их стратегическая значимость оценивается как невысокая, см. рис. 3, блок 7. Такие проекты можно рассматривать как потенциально благоприятные возможности.

Аналогичные рассуждения могут быть приняты во внимание и при принятии решений относительно включения в итоговый вариант стратегического портфеля тех проектов, которые имеют среднюю приоритетность как относительно стратегической значимости, так и относительно финансовой эффективности. 


\section{3. ВЫВОДЫ}

Предложены методические основы к разработке моделей комплексного обоснования приоритетности проектов стратегического портфеля систем перевозок в организациях ТДГАТ, которая предусматривает оценивание проектов по параметрам стратегической значимости и финансовой эффективности.

\section{ЛИТЕРАТУРА}

[1] Бальцер Д. Планирование эксперимента в исследовании технологических процессов. М.: Мир, 1977.

[2] Бушуев С.Д. Управление проектами: основы профессиональных знаний и система оценки компетентности проектных менеджеров. - К.: ІРІДІУМ, 2006. - 208 с.

[3] Воркут Т.А. Проектний аналіз. - Навчальний посібник для студентів вищих навчальних закладів, що навчаються за напрямом «Транспортні технології». - Київ: Український центр духовної культури, 2000. - 440 с.

[4] Нивен П. Р. Диагностика сбалансированной системы показателей. Пер с англ. Дніпропетровськ: Баланс Бизнес Букс, 2006. - 256 с.

[5] Тернер Дж.Р. Руководство по проектно-ориентированному управлению. Пер.с англ. / Под общ. Ред. В.И. Воропаева. - М.: ИД «Гребенников», 2007. - 552 с.

[6] Шишкин Е.В. Математические методы и модели в управлении. - М.: Дело, 2000. -438 с.

[7] Dasgupta P.S. Guidelines for Project Evaluation (UNIDO Guidelines). - New York: United Nations, 1972.

[8] Saaty N.T. The analytic hierarchy process. - New York: Mc Graw-Hill, 1984. - 374 p.

\section{CREATION OF STRATEGIC PORTFOLIO OF PROJECTS IN ORGANIZA- TIONS OF TERMINAL DELIVERY OF LOADS BY ROAD TRANSPORT}

In the article there were presented the methodical bases of the model development of complex ground of project priority on strategic portfolio of the systems of transportations in organizations of terminal delivery of loads by a road transport, that estimates the projects assessment according to the parameters of strategic meaningfulness and financial efficiency.

DOI: $10.7862 /$ rz.2012.zim.32 